\title{
Experimental Evaluation of Multiple Antenna Techniques for Remote Sensing of Physiological Motion
}

\author{
Dragan Samardzija ${ }^{1}$, Byung-Kwon Park ${ }^{2}$, Olga Boric-Lubecke ${ }^{2}$, Victor Lubecke ${ }^{2}$, Anders Host-Madsen ${ }^{2}$, \\ and Theodore Sizer ${ }^{1}$ \\ ${ }^{1}$ Alcatel-Lucent, Bell Laboratories,791 Holmdel-Keyport Road, New Jersey, 07733, \\ USA, Email: dragan, ts2@bell-labs.com, \\ ${ }^{2}$ University of Hawaii at Manoa, 2540 Dole St, Honolulu, 96822, \\ USA, E-mail: byungp, olgabl, lubecke, madsen@ hawaii.edu.
}

\begin{abstract}
In this paper we present a wireless multiple antenna experimental system that is used for remote sensing of physiological motion. We introduce a novel detection algorithm that exploits multiple antennas. The algorithm is based on the analysis of the received signal power spectrum, where a unique cost is assigned to each spectral component. The assigned cost approximates a probability that the measured spectral component is a result of the target's motion. Furthermore, we experimentally evaluate its performance demonstrating the feasibility of the detection of the breathing signal, and demonstrating significant gains due to the application of multiple transmit and receive antennas.
\end{abstract}

Index Terms - Biomedical monitoring, biomedical signal detection, MIMO, remote sensing, physiological motion.

\section{INTRODUCTION}

Doppler radar remote sensing of cardio and pulmonary activity (i.e. physiological motion) has been a promising technique for health monitoring and security applications [1, 2]. Single antenna systems have been described in $[3,4]$. In these systems, analog signal conditioning was used to amplify baseband output, remove the direct component (DC), and avoid aliasing, while digital signal processing was used to determine heart and breathing rates. In this paper we propose to use multiple antenna multiple-input multiple-output (MIMO) systems and techniques to enhance the detection of cardio-pulmonary signals. Some prior work on this topic can be found in [5-7].

MIMO systems take advantage of random scattering of radio signals between transmit and receive antennas. This scattering is known as multipath, since it results in multiple copies of the transmitted signal arriving at the receivers via different scattered paths. In conventional wireless systems, multipath could result in a destructive interference, and is thus considered undesirable. However, MIMO systems exploit multipath by treating scattering paths as separate parallel subchannels [8]. Similarly, the remote sensing of physiological motion can exploit the multiple copies of the transmitted signal.
In this paper we describe a multiple antenna experimental system that is used for the remote sensing. Furthermore we introduce a novel detection algorithm that exploits multiple antennas. The algorithm is based on the analysis of the received signal power spectrum between each transmit and receive antenna. The measured received power spectrum is compared to a reference power spectrum. A unique cost is assigned to each spectral component. The assigned cost approximates a probability that the measured spectral component is a result of the target's motion. The costs are combined among multiple receive and transmit antennas yielding the decision statistics. Significant gains due to the application of multiple antennas are demonstrated.

In Section II and III we present the MIMO experimental system and algorithm details. The experimental results are given in Section IV, and we conclude in Section V.

\section{EXPERIMENTAL SYSTEM}

The experimental system is based on a general purpose wireless multiple antenna testbed. It consists of transmit and receive antenna array each with $N_{t x}$ and $N_{r x}$ antennas, respectively. In this particular case $N_{t x}=2$ and $N_{r x}=4$.
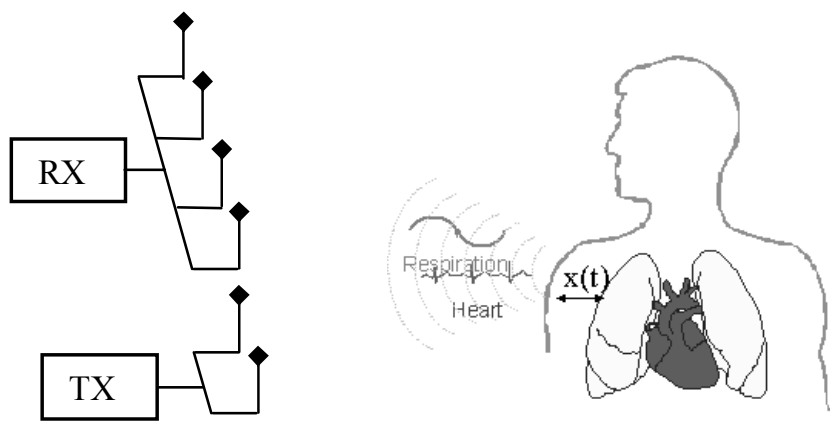

Fig. 1: The experimental system consists of $N_{t x}=2$ transmit antennas and $N_{r x}=4$ receive antennas, with the target of observation in the antenna field.

Each transmit antenna is connected to a general-purpose signal generator transmitting a unique continuous wave $(\mathrm{CW})$. 
Transmit antenna 1 and 2 are set to $f_{1}=2.4 \mathrm{GHz}+1 \mathrm{KHz}$, and $f_{2}=2.4 \mathrm{GHz}-1 \mathrm{KHz}$, respectively. The receiver is build using off the shelf components in heterodyne architecture, two-step down-conversion heterodyne architecture with intermediate frequencies (IF) of $140 \mathrm{MHz}$ and $10 \mathrm{MHz}$. High-speed analogto-digital (AD) conversion digitized the IF signal. The signal is further digitally down-converted to the baseband with the 14-bit resolution. The baseband samples are then stored to a PC hard-drive for further analysis and post-processing. The transmitter and receiver share a common synchronization source. Specifically, their local oscillators are driven by a common $10 \mathrm{MHz}$ reference.

\section{ALGORITHM}

In this section we present details of the multiple antenna algorithm that is used to detect certain parameters of physiological motion. Specifically, we determine spectral components of the received signal that correspond to the target's motion. Basic features of the algorithm are outlined in the following.

- The algorithm is based on the analysis of the received signal power spectrum between each transmit and receive antenna.

- A spatial zero-forcing filter is applied so that the DC is removed from the measured received signal.

- The measured received power spectrum is compared against a reference power spectrum. Based on how different each spectral component of the measured and reference power spectrum is, a unique cost is assigned to each component.

- The assigned cost approximates a probability that the measured spectral component is a result of the target's motion, i.e., it is not due to noise. In other words, higher cost corresponds to a higher probability of the component being due to the target's motion.

- The costs are combined among multiple receive and transmit antennas yielding the decision statistics.

More details are given in the following.

\section{A. Power Spectrum Reference}

During the reference measurements each transmitter and receive are wired. For each transmit power level, 100 independent measurements are taken, corresponding to $T=$ $6.144 \mathrm{sec}$, yielding the frequency resolution of $\Delta f=0.1628$ $\mathrm{Hz}$. The sampling rate is $f_{\text {smp }}=15 \mathrm{KHz}$. The power spectrum is determined by the conventional FFT.

Using the reference measurements, the maximum and average power spectrum is found per each component. Fig. 2 presents the maximum and average reference power spectrum between one transmit and four receive branches. In Fig. 2 we note a presence of the phase noise (a typical skirt-like shape around the DC), as well as, some spurious noise (e.g., at 1.8 $\mathrm{Hz}$ and $3.6 \mathrm{~Hz}$ ).

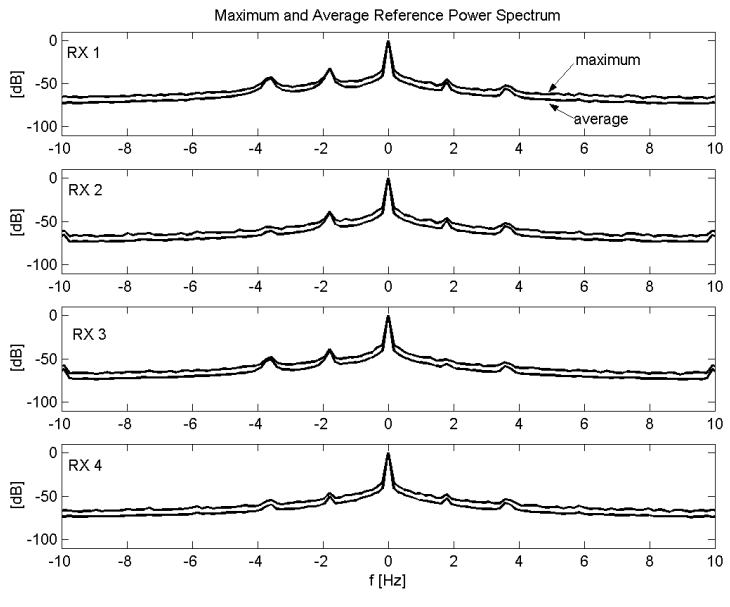

Fig. 2: The maximum and average reference power spectrum between one transmit and four receive branches.

\section{B. Spatial DC Notching}

The received signal vector is

$$
\mathbf{r}_{m}(k)=\left[r_{1, m}(k) \ldots r_{N_{r x}, m}(k)\right]^{\mathrm{T}}
$$

where $r_{n, m}(k)$ is the signal transmitted by transmit antenna $m$ and received by receive antenna $n$ at the time instance $k$. The vector of the DC coefficients is

$$
\mathbf{v}_{m}=\left[u_{1, m} \ldots u_{N_{r x}, m}\right]^{\mathrm{T}}
$$

where $u_{n, m}=\mathrm{E}\left[r_{n, m}(k)\right]$. It is normalized as

$$
\overline{\mathbf{v}}_{m}=\frac{\mathbf{v}_{m}}{\sqrt{\left|\mathbf{v}_{m}\right|^{2}}} .
$$

The DC is removed from the received vector $\mathbf{r}_{m}(k)$ as

$$
\overline{\mathbf{r}}_{m}(k)=\mathbf{r}_{m}(k)-\left(\overline{\mathbf{v}}_{m}{ }^{\mathrm{H}} \mathbf{r}_{m}(k)\right) \overline{\mathbf{v}}_{m}
$$

An example of the measured spectrum before and after the DC notching is given in Fig. 3 . 


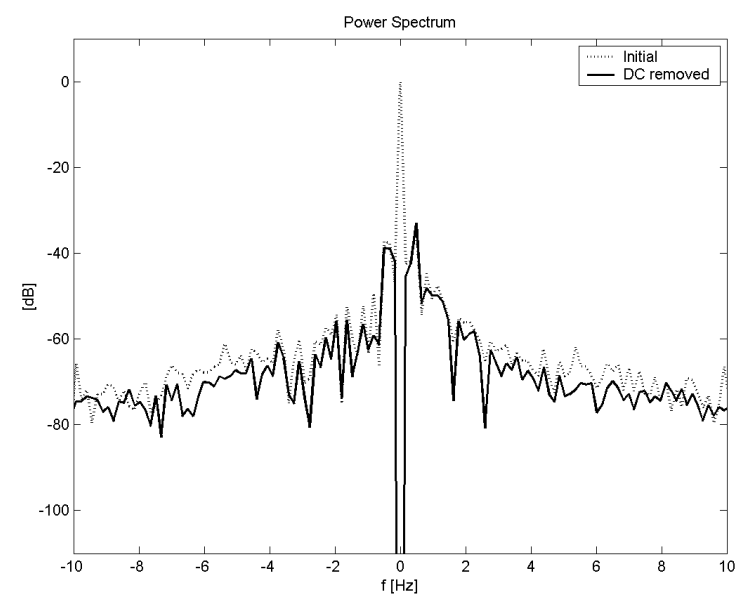

Fig. 3: An example of the measured power spectrum before and after the DC notching (for the signal that is transmitted by transmit antenna 1 and received by receive antenna 1 ).

\section{Cost Function}

In the following, $S_{n, m}(f)$ denotes the measured power spectrum of the signal that is transmitted by transmit antenna $m$ and received by receive antenna $n$, for the frequency component $f$. Similarly, $S_{n, m}^{\max }(f)$ denotes the maximum reference power spectrum, which is previously described. The cost functions that we use in this study is

$$
c_{n, m}(f)=\left\{\begin{array}{lc}
0.1\left(\frac{S_{n, m}(f)}{S_{n, m}^{\max }(f)}\right)^{2} & \text { for } S_{n, m}(f) \leq S_{n, m}^{\max }(f) \\
1-0.9\left(\frac{S_{n, m}^{\max }(f)}{S_{n, m}(f)}\right)^{2} & \text { else }
\end{array},\right.
$$

The cost function is design so that is has similar properties as a probability mass function. For example, it is non-negative, and does not exceed 1. Note that for large values of $S_{n, m}(f)$ relative to the reference, the cost functions is approaching the maximum value 1 . This actually quantifies a case when the measured power spectrum is significantly stronger than what was experienced during the reference measurements. In other words, the spectral components with high values of the cost function are most probably not due to the noise, but rather due to a motion of the target in the antenna field.

The cost function may be designed to have different characteristics. This is a subject of our future work.

\section{Multiple Antenna Decision Statistics}

Considering similarities between the cost function and probability that a particular spectral component is due to the target's motion, the joint cost function for every transmit and receive antenna pair is

$$
c(f)=\prod_{m=1}^{N_{t x}} \prod_{n=1}^{N_{t x}} c_{n, m}(f) .
$$

The decision statistics is obtained by normalizing the above joint cost function as

$$
D(f)=\frac{c(f)}{\sum_{f_{i}=-F_{\max }}^{-F_{\max }} c\left(f_{i}\right)} .
$$

\section{EXPERIMENTAL RESULTS}

The target is typically positioned within 1 to 2 meters from the antenna arrays. It is facing both transmit and receive antenna array. The transmit antennas are set $2 \lambda$ apart (where $\lambda$ is the wavelength), while the receive antennas are set $\lambda$ apart.

In Fig. 4 we present the measured power spectrum with a person sitting in front of the antenna arrays. The measured power spectrum is compared against the maximum reference power spectrum. Based on the measurements, the dominant spectral component is at $0.4878 \mathrm{~Hz}$. It corresponds to the person's pulmonary activity (i.e., breathing).

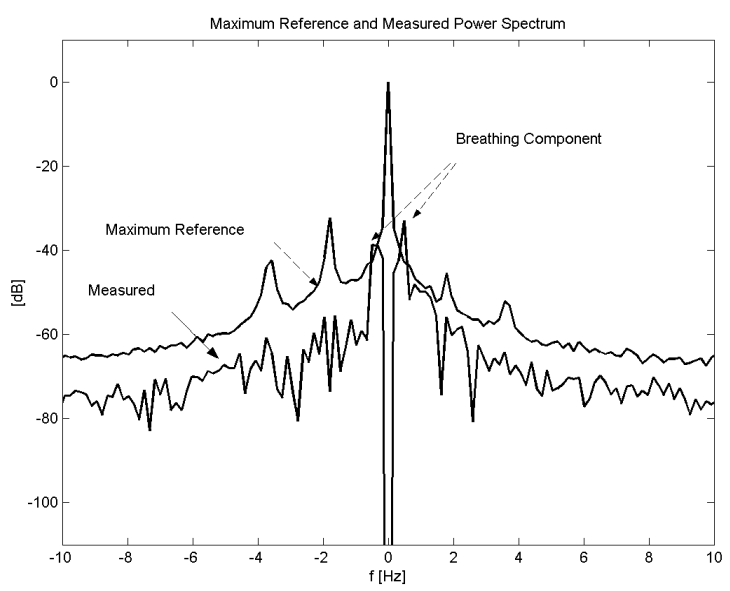

Fig. 4: The measured and maximum reference power spectrum with a person sitting in front of the antenna array (for the signal that is transmitted by transmit antenna 1 and received by receive antenna 1).

In Fig. 5 the decision statistics $D(f)$ corresponding to the above experimental scenario is presented for $N_{t x}=2, N_{r x}=4$, and $F_{\max }=20 \mathrm{~Hz}$. From the results we note that the spectral component at $0.4878 \mathrm{~Hz}$ is most probably due to a periodic motion of the target, i.e., the person's pulmonary activity. 
Independent measurements of the breathing rate using a piezoelectric chest belt point to the same result.

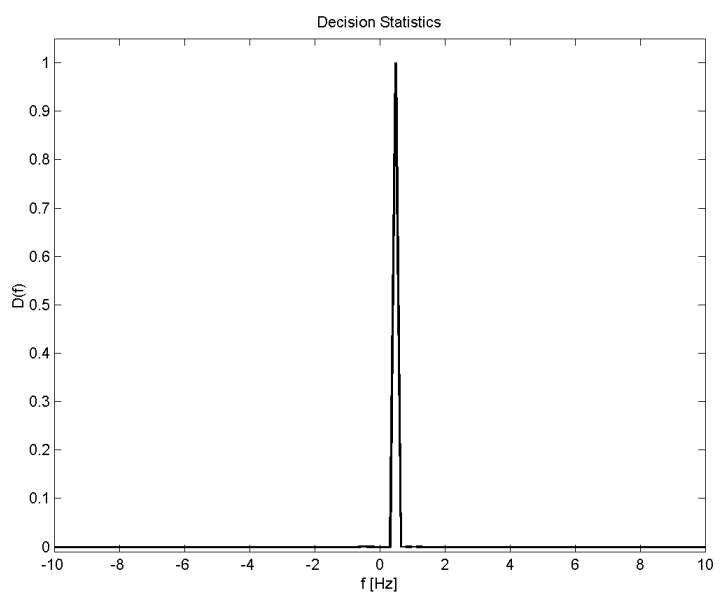

Fig. 5: The decision statistics $D(f)$ for a person sitting in front of the antenna array, $N_{t x}=2, N_{r x}=4, F_{\max }=20 \mathrm{~Hz}$.

In Fig. 6 the decision statistics $D(f=0.4878 \mathrm{~Hz})$ is presented as a function of the number of transmit and receive antennas. $D(f=0.4878 \mathrm{~Hz})$ increases with the number of antennas. This clearly demonstrates the benefits of multiple antennas.

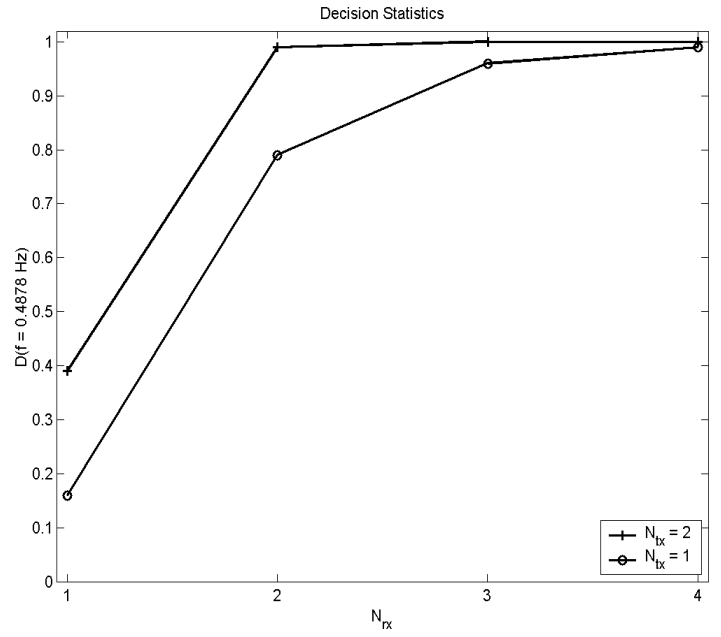

Fig. 6: The decision statistics $D(f=0.4878 \mathrm{~Hz})$ for different number of transmit and receive antennas.

We also determined the decision statistics for a case when no target is placed in front of the antenna arrays. The results correspond to $N_{t x}=2, N_{r x}=4$, and $F_{\max }=20 \mathrm{~Hz}$. For the frequencies of interest, the decision statistics is very low (practically zero), indicating that no motion is present. This further demonstrates ability of the algorithm to distinguish cases with and without the target being present.

\section{CONCLUSIONS}

In this paper we have introduced the detection algorithm that exploits multiple antennas. The algorithm is based on the analysis of the received signal power spectrum. A unique cost is assigned to each spectral component. The assigned cost approximates a probability that the measured spectral component is a result of the target's motion. The costs are combined among multiple receive and transmit antennas yielding the decision statistics. In addition, we have described spatial zero-forcing filtering that is used to remove the DC. We have performed a number of experiments that are used to assess the algorithm performance.

We have considered the performance as a function of the number of transmit and received antennas. Significant gains due to the application of multiple antennas are demonstrated. Furthermore, we have demonstrated the ability of the algorithm to distinguish cases with and without the target being present.

Our future work will address the design of the cost function as well as improvements to the multiple antenna testbed. Specifically, increasing the AD resolution and introduction of the analog DC filtering are the subject of our future work.

\section{ACKNOWLEDGEMENT}

This research was partially sponsored by the NSF under contract ECS0428975.

\section{REFERENCES}

[1] J. C. Lin, "Microwave sensing of physiological movement and volume change: a review," Bioelectromagnetics, vol. 13, pp. 557-565, 1992.

[2] V. M. Lubecke, O. Boric-Lubecke, G. Awater, P.-W. Ong, P. Gammel, R.H. Yan, J. C. Lin, "Remote sensing of vital signs with telecommunications signals," World Congress on Medical Physics and Biomedical Engineering (WC2000), Chicago IL, July 2000.

[3] A. D. Droitcour, O. Boric-Lubecke, V. M. Lubecke and J. Lin, "0.25 mm CMOS and BiCMOS single-chip direct-conversion Doppler radars for remote sensing of vital signs," ISSCC Digest, vol.1 pp.348-348, 2002.

[4] V. M. Lubecke, O. Boric-Lubecke, and E. Beck, "A compact low-cost addon module for Doppler radar sensing of vital signs using a wireless communications terminal," MTT-S IMS Digest, 2002.

[5] D. Samardzija, O. Boric-Lubecke, A. Host-Madsen, V. M. Lubecke, T. Sizer, A. D. Droitcour, and G. T. A. Kovacs, "Applications of MIMO techniques to sensing of cardiopulmonary activity," IEEE/ACES Conference on Wireless Communications and Applied Computational Electromagnetics, 2005.

[6] O. Boric-Lubecke, V. M. Lubecke, A. Host-Madsen, D. Samardzija, K. Cheung, "Doppler radar sensing of multiple subjects in single and multiple antenna systems," 7th International Conference on Telecommunications in Modern Satellite, Cable and Broadcasting Services (TELSIKS), Nis 2005.

[7] Q. Zhou, J. Liu, A. Host-Madsen, O. Boric-Lubecke, V. Lubecke, "Detection of multiple heartbeats using Doppler radar," IEEE ICASSP, Honolulu 2007.

[8] J. Foschini, "Layered space-time architecture for wireless communication in a fading environment when using multiple antennas," Bell Labs Technical Journal, vol. 1, no. 2, Autumn 1996, pp 41-59. 